\title{
Anesthetic considerations of Joubert syndrome in patients with mitochondrial disease - A case report -
}

\section{Jeong Yeon Kim', Koun Jeong' ${ }^{1}$, Ki Seob Han ${ }^{1}$, Ji Eun Park', Mun Gyu Kim², and Mi Roung Jun ${ }^{1}$}

'Department of Anesthesiology and Pain Medicine, Samsung Changwon Hospital, Sungkyunkwan University School of Medicine, Changwon, ${ }^{2}$ Department of Anesthesiology and Pain Medicine, Soonchunhyang University Seoul Hospital, Seoul, Korea
Received November 26, 2020

Revised February 3, 2021

Accepted February 5, 2021

\section{Corresponding author}

Mi Roung Jun, M.D.

Department of Anesthesiology and Pain Medicine, Samsung Changwon Hospital, Sungkyunkwan University School of Medicine, 158 Paryong-ro, Masanhoewon-gu, Changwon 51353, Korea

Tel: 82-55-233-6045

Fax: 82-55-233-5524

E-mail: ane2017@naver.com
Background: Joubert syndrome and mitochondrial disease are rare congenital diseases in which a wide range of symptoms affects multiple organs. Patients with these diseases present characteristic symptoms related to the musculoskeletal, respiratory, and neurological systems, which make it difficult for anesthesiologists to manage the patient's airway and choose appropriate anesthetic drugs.

Case: A 13-year-old male patient with Joubert syndrome and mitochondrial disease underwent elective surgery to insert a continuous ambulatory peritoneal dialysis catheter. Anesthesia was induced and maintained with propofol, remifentanil, and rocuronium. An l-gel was inserted to secure the airway; however, the fitting did not work properly, so the patient was intubated. The operation was completed without any major problems, and the intubated patient was transferred to the intensive care unit.

Conclusions: Anesthesiologists should determine the method of anesthesia and prepare for unintended complications based on a full understanding of these congenital diseases.

Keywords: Airway management; Anesthesia, intravenous; Cerebellar diseases; Mitochondrial disease.
Joubert syndrome (JS) is a rare autosomal recessive disorder, characterized by hypotonia, ataxia, episodic hyperventilation, abnormal eye movement, and psychomotor retardation [1]. Some of these clinical features of JS correspond to those of patients with mitochondrial disease (MD). MD manifests symptoms based on the ratio of normal mitochondrial DNA to mutated mitochondrial DNA [2]. Mitochondria are an important survival-related component, and the range of diseases that can be manifested by dysfunction in mitochondrial metabolic pathways is very wide. Anesthesia management for patients with these congenital disorders and coping with unexpected complications during surgery is a challenge for anesthesiologists. It is important to thoroughly identify the patient's condition and carefully select and prepare the best anesthesia method. Here, we present the anesthetic care of a patient who had been diagnosed with JS and MD.

\section{CASE REPORT}

Written informed consent was obtained for publication of this report.

This is an Open Access article distributed under the terms of the Creative Commons Attribution Non-Commercial License (http://creativecommons.org/licenses/by-nc/4.0) which permits unrestricted non-commercial use, distribution, and reproduction in any medium, provided the original work is properly cited.

Copyright (C) the Korean Society of Anesthesiologists, 2021 
A 13-year-old male patient was scheduled for elective insertion of a continuous ambulatory peritoneal dialysis (CAPD) catheter. The admission height and weight were $135 \mathrm{~cm}$ and $31 \mathrm{~kg}$, respectively. The patient was born at 40 weeks by normal vaginal delivery and was admitted to the neonatal intensive care unit for 10 days because of sepsis. MD was diagnosed at 12 months of age and then JS was diagnosed during outpatient observation. The patient started hemodialysis due to end-stage renal disease at the age of 8 years and was hospitalized at age 10 years with intracranial hemorrhage due to high blood pressure. The patient was admitted at age 11 years for uncontrolled hypertension and a change in the permanent catheter, but hyperkalemia-induced cardiac arrest occurred. The patient returned to spontaneous circulation after $1 \mathrm{~h}$ of cardiopulmonary resuscitation (CPR) and was transferred to the intensive care unit (ICU) to receive post-CPR care for 2 weeks. Subsequently, the patient was hospitalized and discharged repeatedly due to heart, lung, and kidney problems. He underwent regular hemodialysis; however, hemoglobin level and blood pressure continued to fall and could not be controlled. Therefore, the patient was scheduled to switch to peritoneal dialysis.

In terms of mental status, the patient was stuporous during pre-anesthetic assessment (Glasgow coma scale [GCS] score 11). He was bed-ridden, with a percutaneous endoscopic gastrostomy tube that was inserted in the surgical ICU (SICU). Hypotension was managed by infusion of norepinephrine (0.07-0.19 $\mu \mathrm{g} / \mathrm{kg} / \mathrm{min})$. His mean blood pressure (MBP) and his heart rate (HR) were controlled at 90-106 mmHg and 95-107 beats/min, respectively, and checked every $30 \mathrm{~min}$. His breathing was shallow; however, $100 \%$ oxygen saturation was maintained on room air. We reviewed all of the records, including previous hospital charts, but the patient had never undergone surgery, and we found no accurate information about his airway. The patient's jaw was small, his mouth was slightly protruding, and he had a short neck with reduced cervical mobility. His airway could not be evaluated properly due to the poor opening of his mouth because of the rigidity of his jaw; thus, we decided to perform a reassessment at the time of anesthesia induction on the day of surgery. Three anesthesiologists decided to participate in induction because this was a rare case that we had not previously experienced in our hospital. We decided to have the following instruments in place for airway management: oral airway (size 1 and 2), stylets, a Macintosh direct laryngoscope (curved blade siz- es 2 and 3), a video-laryngoscope (McGrath, Aircraft Medical, UK and C-MAC, Karl Storz, Germany), a fiberoptic bronchoscope (Olympus Optical, Japan), and a supraglottic airway device (SAD; I-gel, Intersurgical Ltd., UK). We selected an endotracheal tube (ETT) according to age based on height and weight and prepared 5.0- and 5.5-mm tubes with cuffs.

Blood sugar before leaving for the operating room was $132 \mathrm{mg} / \mathrm{dl}$. In the operating room, routine standard monitors were attached (noninvasive blood pressure [NIBP], electrocardiography, pulse oximetry); the initial NIBP and HR were 120/90 $\mathrm{mmHg}$ and 105 beats/min, respectively, with a norepinephrine infusion of $0.07 \mu \mathrm{g} / \mathrm{kg} / \mathrm{min}$. Invasive blood pressure monitoring was not performed and the surgery time was expected to be short; thus, the operation proceeded after preparing for left radial artery cannulation, if necessary. The degree of neuromuscular blockade, assessed using train-of-four (TOF) stimulation, was monitored using an IntelliVue NMT monitor (Philips, the Netherlands), and bispectral index (BIS) monitoring (Covidien, USA) was performed to assess the depth of anesthesia. The initial BIS was 71. Anesthesia was started with propofol (1 $\mathrm{mg} / \mathrm{kg})$, remifentanil $(0.05 \mu \mathrm{g} / \mathrm{kg} / \mathrm{min})$, and rocuronium $(0.4 \mathrm{mg} / \mathrm{kg})$ following preoxygenation. Intravenous propofol and remifentanil infusions were started at 77-102 $\mu \mathrm{g} /$ $\mathrm{kg} / \mathrm{min}$ and $0.05-0.1 \mu \mathrm{g} / \mathrm{kg} / \mathrm{min}$, respectively, to maintain anesthesia (BIS 40-60). The patient's jaw was small; however, his palate was not malformed, his tongue did not protrude, and mask ventilation was well maintained. A thirdyear anesthesiology resident tried to insert an I-gel of size 2.5 , which was selected according to the patient's weight. The resident began to insert the I-gel along the hard palate with one hand pressing down on the patient's chin. Resistance was felt while inserting the I-gel, thus he gently pushed the I-gel using the deep rotation method and connected the I-gel to the ventilator. However, air continued to leak, and a sufficient tidal volume was not attained. The attending anesthesiologist determined that the I-gel did not fit properly, and removed and inserted it again using the jaw thrust method with the help of another attending anesthesiologist. Nevertheless, air continued to leak, and the I-gel was removed, as it had become malpositioned. The trachea was secured with a 5.0-mm ETT without difficulty using a McGrath (blade size 2) by the attending anesthesiologist, and the Cormack-Lehane grade was 1. A pilot balloon was inflated with room air to a cuff pressure of $18 \mathrm{~cm}$ $\mathrm{H}_{2} \mathrm{O}$ as measured by a manometer (VBM, Germany). Lung 
sounds had decreased on the left side and coarse breath sounds were auscultated over both lower lung fields. The ETT was fixed at $15 \mathrm{~cm}$ from the lower incisor teeth. Mechanical ventilation was performed with a 50:50 air/oxygen mixture, a tidal volume of $6-8 \mathrm{ml} / \mathrm{kg}$, a respiratory rate of 16-18 breaths/min, a maximum peak airway pressure of 11-14 $\mathrm{cmH}_{2} \mathrm{O}$, and an inspiratory/expiratory time ratio of 1:2. End-tidal carbon dioxide and oxygen saturation were maintained at $35-39 \mathrm{mmHg}$ and $100 \%$ during the surgery, respectively. The operation lasted $45 \mathrm{~min}$, and the patient's hemodynamics were stable during the procedure. The MBP and HR were controlled at 73-103 mmHg and 105130 beats/min, respectively, with a norepinephrine (0.03$0.07 \mu \mathrm{g} / \mathrm{kg} / \mathrm{min}$ ) infusion. The TOF count was maintained at 1-2 during surgery, and there was no additional injection of muscle relaxants. No additional opioids were used other than remifentanil. At the end of the operation, the patient's spontaneous breathing returned and the BIS and TOF ratio were 73 and $76 \%$, respectively; however, the respiration rate was $<10$ times $/ \mathrm{min}$. The pediatrician wanted to observe the patient's breathing pattern more carefully; thus, after injecting $3 \mathrm{mg}$ midazolam, the patient was transferred to the SICU with the ETT. In the SICU, the patient's mental state was stuporous (GCS score 6), and a ventilator was applied with the following settings: synchronized intermittent mandatory ventilation mode with a $\mathrm{FiO}_{2}$ of 0.25 , tidal volume of $6 \mathrm{ml} / \mathrm{kg}$, respiratory rate of 20 breaths $/ \mathrm{min}$, and pressure support of $13 \mathrm{cmH}_{2} \mathrm{O}$. The MBP and HR were maintained at $105-111 \mathrm{mmHg}$ and $124-137$ beats/min, respectively, with a norepinephrine $(0.07 \mu \mathrm{g} / \mathrm{kg} / \mathrm{min})$ infusion. The trachea was extubated at 1 hour after the patient arrived in the ICU, and no irregular respiratory patterns were observed. The patient exhibited $100 \%$ oxygen saturation with a 2-L oxygen flow applied by nasal cannula. According to the venous blood gas analysis, the venous $\mathrm{pH}$ was 7.30, $\mathrm{PCO}_{2} 53 \mathrm{mmHg}, \mathrm{PO}_{2} 47 \mathrm{mmHg}$, base excess 0.3 $\mathrm{mmol} / \mathrm{L}, \mathrm{SO}_{2} 78 \%$, glucose $130 \mathrm{mg} / \mathrm{L}$, and lactate 0.3 $\mathrm{mmol} / \mathrm{L}$.

\section{DISCUSSION}

JS is a genetic disorder caused by a malformed cerebella vermis. The typical manifestations of JS are hypotonia, ataxia, episodic apnea-hyperpnea, and global developmental delay [1]. JS patients also characteristically have a large tongue, a cleft or highly arched palate, and micrognathia [3]. This disease can also present as a variety of clin- ical features depending on the organs that have been affected; thus, JS increases the risks associated with anesthesia management [1]. Above all, the difficulty with patients with JS is to secure and control the airway due to an abnormal breathing pattern and the differences in facial shape and oral structure [1]. Patients with JS are susceptible to almost any anesthetic, making it difficult to titrate an appropriate dose of the anesthetic agent while maintaining an intact airway for simple procedures.

The focus of the anesthesiologist, in this case, was respiratory management. Inserting a CAPD catheter is a simple procedure that is generally controlled during monitored anesthesia care in our hospital. However, management of the airway was expected to be difficult in this case and general anesthesia was planned. In this case, unfortunately, it was not helpful to review previous hospital records of whether there were issues with the patient's airway. It is important to thoroughly assess any patient feature suggesting a difficult airway [4]. We focused primarily on observing the patient's appearance and the passive movement of the neck due to the poor mouth opening and the inability to respond to commands during the pre-anesthetic evaluation. In addition, indicators, such as upper incisor length, thyromental distance, length of the neck, and neck circumference of the patient would have been helpful for the airway evaluation [4].

Mirghassemi et al. [5] suggested that it is difficult to measure these indicators in pediatric patients; thus, they proposed a method for predicting difficult airways based on measuring and calculating the distances from the lower lip board to the tip of the chin, from the ear tragus to the corner of the mouth, and from the ear lobe to the corner of the mouth. While it is important to prepare advanced airway equipment when a difficult airway is expected, it is also important to have additional anesthesiologists available to assist during emergency situations [4]. We had one anesthesiology resident and two attending anesthesiologists, and each had prepared the advanced airway equipment most familiar to them. As the number of attempts at tracheal intubation increases in patients with a difficult airway, the probability of complications also increases. Thus, for pediatric patients with a difficult airway scheduled a simple procedure that is expected to finish in a short time, as in this case, the use of a SAD may be advantageous [6]. The I-gel is an anatomically shaped SAD that seals the airway without a cuff; thus, it is easy to insert and remains stable. The patient, in this case, had a small jaw, but no 
other major problems were found with the oral structure, so mask ventilation was carried out smoothly; hence, we decided to insert the I-gel. However, the I-gel did not fit properly in two attempts, and eventually, the patient was intubated. Because an I-gel is inserted without direct visualization of the airway, malpositioning is possible, which leads to an incomplete seal, resulting in leakage or obstruction of the airway, eventually leading to problems with ventilation. The anesthesiologist can adjust the depth of the I-gel or choose a different size [7]. Although there was a major leak, in this case, the size of the I-gel was considered appropriate for the patient, so we did not attempt to re-insert a different size. As an alternative, the I-gel was inserted with the jaw thrust method; however, the leak continued, so the anesthesiologist decided to intubate the airway. In addition, post-extubation respiratory problems can occur after positive pressure ventilation during general anesthesia for surgery. It has been recommended that such patients be transferred to the ICU without extubation for controlled ventilation [8]. The patient in this case also developed spontaneous respiration after the surgery; however, the breathing pattern was very weak and slow. The patient was transferred to the ICU while intubated and was extubated after confirming that sufficient tidal volume had been reached.

MD is categorized into various types of mitochondrial genetic mutations and is expressed in a wide range of clinical forms. MD patients usually have myopathies and various clinical manifestations in the central nervous system, heart, gastrointestinal tract, and kidneys [9]. These patients may be vulnerable to situations that can increase metabolic burden, such as prolonged fasting, hypovolemia, and hypothermia, which require attention during the perioperative period [9]. In general, almost all anesthetic agents suppress mitochondrial function, particularly the inhalation anesthetics and propofol [9]. In addition, propofol inhibits mitochondrial metabolism in at least four steps, which is related to propofol infusion syndrome. For this reason, prolonged continuous infusion of propofol in patients with MD should be avoided [8]. Patients with mitochondrial myopathy are likely to develop respiratory failure, so care should be taken when using neuromuscular blocking agents and opioids [9]. Nevertheless, MD patients usually tolerate various anesthetics without major problems [10]. These patients may also have increased susceptibility to malignant hyperthermia [2]; however, this is controversial. Footitt et al. [11] reviewed 58 anesthesia records in pediat- ric patients with MD and found no adverse events, such as malignant hyperthermia or rhabdomyolysis. The Malignant Hyperthermia Association of the United States stated that the susceptibility to malignant hyperthermia does not increase in MD patients; however, they concluded that the selection of an appropriate anesthetic is important depending on the patient's symptoms and type of surgery and that more attention should be paid to the use of succinylcholine [12]. The patient in this case was expected to have a short operation of less than $30 \mathrm{~min}$, so we used propofol and remifentanil, which are relatively easy to titrate with a rapid post-anesthesia recovery [9]. However, a report on MD patients showed delayed recovery after a short propofol infusion and the need for ICU care [13]. The use of less than $200 \mathrm{mg}$ of propofol might have affected the recovery from anesthesia of the patient in our case.

Patients who have been diagnosed with both mitochondrial dysfunction and JS, such as our patient, have been reported, but they are very rare [14]. These diseases are characterized by different ranges of symptoms depending on the organs that are affected. However, symptoms in the musculoskeletal, respiratory, and neurological systems can be similar in both

diseases and are important considerations during anesthesia management $[1,10]$. Several studies have shown that JS and MD patients can tolerate most of the commonly use anesthetics [15].

In summary, it may be difficult to manage anesthesia in pediatric patients with more than one congenital condition, and the characteristics of each abnormality will need to be well understood. In this case, we focused more on MD when selecting the anesthetics and focused on JS for airway management. In addition, the difficulty and expected time of the scheduled surgery were also considered. There is generally no clear anesthesia indication for patients with several congenital diseases. It would be ideal for the anesthesiologist to properly apply the carefully chosen anesthesia technique under surveillance monitoring, and preparation for an emergency is also important.

\section{CONFLICTS OF INTEREST}

No potential conflict of interest relevant to this article was reported. 


\section{DATA AVAILABILITY STATEMENT}

Data sharing not applicable to this article as no datasets were generated or analyzed during the current study.

\section{AUTHOR CONTRIBUTIONS}

Conceptualization: Jeong Yeon Kim, Mi Roung Jun, Koun Jeong. Data curation: Ki Seob Han, Ji Eun Park. Writing original draft: Jeong Yeon Kim, Mi Roung Jun. Writing - review \& editing: Mi Roung Jun, Koun Jeong, Mun Gyu Kim. Supervision: Mun Gyu Kim.

\section{ORCID}

Jeong Yeon Kim, https://orcid.org/0000-0003-0676-723X

Koun Jeong, https://orcid.org/0000-0002-2987-3759

Ki Seob Han, https://orcid.org/0000-0001-7820-7771

Ji Eun Park, https://orcid.org/0000-0002-1025-793X

Mun Gyu Kim, https://orcid.org/0000-0003-1992-8215

Mi Roung Jun, https://orcid.org/0000-0003-0330-9102

\section{REFERENCES}

1. Brancati F, Dallapiccola B, Valente EM. Joubert syndrome and related disorders. Orphanet J Rare Dis 2010; 5: 20.

2. Muravchick S. Clinical implications of mitochondrial disease. Adv Drug Deliv Rev 2008; 60: 1553-60.

3. Kumandas S, Akcakus M, Coskun A, Gumus H. Joubert syndrome: review and report of seven new cases. Eur J Neurol 2004; 11: $505-10$

4. Krishna SG, Bryant JF, Tobias JD. Management of the difficult airway in the pediatric patient. J Pediatr Intensive Care 2018; 7: 115-25.

5. Mirghassemi A, Soltani AE, Abtahi M. Evaluation of laryngoscopic views and related influencing factors in a pediatric pop- ulation. Paediatr Anaesth 2011; 21: 663-7.

6. Jagannathan N, Sohn L, Fiadjoe JE. Paediatric difficult airway management: what every anaesthetist should know! Br J Anaesth 2016; 117 Suppl 1: i3-5.

7. Van Zundert AA, Kumar CM, Van Zundert TC. Malpositioning of supraglottic airway devices: preventive and corrective strategies. Br J Anaesth 2016; 116: 579-82.

8. Habre W, Sims C, D'Souza M. Anaesthetic management of children with Joubert syndrome. Paediatr Anaesth 1997; 7: 251-3.

9. Niezgoda J, Morgan PG. Anesthetic considerations in patients with mitochondrial defects. Paediatr Anaesth 2013; 23: 785-93.

10. Driessen J, Willems S, Dercksen S, Giele J, van der Staak F, Smeitink J. Anesthesia-related morbidity and mortality after surgery for muscle biopsy in children with mitochondrial defects. Paediatr Anaesth 2007; 17: 16-21.

11. Footitt EJ, Sinha MD, Raiman JA, Dhawan A, Moganasundram S, Champion MP. Mitochondrial disorders and general anaesthesia: a case series and review. Br J Anaesth 2008; 100: 436-41.

12. Malignant Hyperthermia Association of the United States. Does mitochondrial myopathy (MM) increase an individual's susceptibility to malignant hyperthermia (MH)? Malignant Hyperthermia Association of the United States [Internet]. 2012 [cited 2021 Jan 29]. Available from https://www.mhaus.org/ healthcare-professionals/mhaus-recommendations/does-mitochondrial-myopathy-mm-increase-an-individuals-susceptibility-to-malignant-hyperthermia-mh/.

13. Farag E, Deboer G, Cohen BH, Niezgoda J. Metabolic acidosis due to propofol infusion. Anesthesiology 2005; 102: 697-8. author reply 698-9.

14. Morava E, Dinopoulos A, Kroes HY, Rodenburg RJ, van Bokhoven $\mathrm{H}$, van den Heuvel LP, et al. Mitochondrial dysfunction in a patient with Joubert syndrome. Neuropediatrics 2005; 36: 214-7.

15. Kloka J, Blum LV, Piekarski F, Zacharowski K, Raimann FJ. Total intravenous anesthesia in Joubert syndrome patient for otorhinolaryngology surgery: a case report and mini review of the literature. Am J Case Rep 2020; 21: e923018. 